\title{
Selective Population of Edge States in a 2D Topological Band System
}

\author{
Bogdan Galilo, ${ }^{1}$ Derek K. K. Lee, ${ }^{2}$ and Ryan Barnett ${ }^{1}$ \\ ${ }^{1}$ Department of Mathematics and ${ }^{2}$ Blackett Laboratory, \\ Imperial College London, London SW7 2AZ, United Kingdom
}

(Dated: May 3, 2022)

\begin{abstract}
We consider a system of interacting spin-one atoms in a hexagonal lattice under the presence of a synthetic gauge field. Quenching the quadratic Zeeman field is shown to lead to a dynamical instability of the edge modes. This, in turn, leads to a spin current along the boundary of the system which grows exponentially fast in time following the quench. Tuning the magnitude of the quench can be used to selectively populate edge modes of different momenta. Implications of the intrinsic symmetries of Hamiltonian on the dynamics are discussed. The results hold for atoms with both antiferromagnetic and ferromagnetic interactions.
\end{abstract}

PACS numbers: 67.85.d, 03.75.b, 37.10.Jk, 71.45.Lr

Topological Bloch bands and their concomitant protected edge modes play important physical roles in several solid-state materials including quantum Hall systems [1] and topological insulators/superconductors [2,3]. Recent years have experienced remarkable progress in engineering systems which possess topological Bloch bands as a result of induced effective gauge fields. Effective gauge fields have been realized in systems of ultracold atoms through mechanical rotation, optical lattice 'shaking', and laser-assisted tunneling (see [4] for a review). Recent milestones include the experimental realization of the Rice-Mele [5], Hofstadter [6-8], and Haldane [9] models in optical lattice systems. The emerging field of topological photonic lattices offers a separate and complementary system where topological Bloch bands have also recently been realized [10-13]. Most of the experimental systems with ultracold atoms involve temperatures for which the particle dynamics can be accurately described by non-interacting theories. However, as experimental techniques are refined and quantum degeneracy is reached, interactions and particle statistics will play an essential role in the physics of these systems.

Of central interest in each of these models is the presence of topologically protected edge modes. The physical consequence of edge modes for a degenerate Fermi gas in a topological band is clear: if the Fermi energy resides within the bulk band gap, the near-equilibrium dynamics will be described by degrees of freedom localized along the edge of the system. On the other hand, for bosonic systems, particles will generally condense into the lowest bulk band, leaving the higher-energy edge states largely unimportant for the dynamics of the system.

In this work, we propose a scheme to bring to the fore the role of edge states in the dynamics of bosons in a prototypical two-dimensional topological band system. In particular, we describe how a quantum quench of an interacting spinor generalization of the well-known Kane-Mele model [14] can result in an exponentially fast growth in the population of edge (but not bulk) states. This will be exhibited as an exponential growth of the spin current along the boundary of the system. Furthermore, the momenta of these edge states can be selected by tuning the magnitude of the quench.

Before proceeding, we briefly comment on previous related work. Probing the topology of band systems through a quench has received considerable attention in the recent theoretical literature (e.g. [15-22]). One of us [23] has described the exponentially fast population of an edge mode in perhaps the simplest topological system, the Su-Schrieffer-Heeger (SSH) model [24], by preparing a bosonic gas in an excited spatial mode. A photonic version of the SSH model was in fact realized [25] where on-site absorption was shown to lead to fast population of edge states [26]. Quadratic fermionic hamiltonians have been previously classified in terms of their symmetries [27, 28]. Much less work, however, has been carried out for bosonic systems. An expression for the Chern number, accounting for the symplectic transformation properties of bosonic systems was obtained in [29]. In [30], the topology of real Bogoliubov excitations in inversionsymmetric lattices was analyzed. However, to our knowledge, a classification of quadratic bosonic systems allowing for dynamical instabilities does not presently exist.

The Haldane model [31] was realized in an ultracold atomic system by the Esslinger group [9]. This system involves atoms on a hexagonal optical lattice where spin-orbit coupling is induced through shaking. In this work, we consider a spin-one version of this system where atoms experience spin-orbit coupling proportional to the $z$-component of their spins. In particular, we introduce the following generalization of the Kane-Mele model [14]:

$$
\hat{\mathcal{H}}_{\mathrm{S} 1 \mathrm{KM}}=-w \sum_{\langle i j\rangle} \hat{\boldsymbol{\Phi}}_{i}^{\dagger} \hat{\boldsymbol{\Phi}}_{j}+i \lambda \sum_{\langle\langle i j\rangle\rangle} \nu_{i j} \hat{\boldsymbol{\Phi}}_{i}^{\dagger} S_{z} \hat{\boldsymbol{\Phi}}_{j}
$$

where $\hat{\boldsymbol{\Phi}}_{i}=\left(\hat{\Phi}_{i, 1}, \hat{\Phi}_{i, 0}, \hat{\Phi}_{i,-1}\right)^{T}$ is a vector composed of bosonic annihilation operators at site $i$ for each spin component and we denote the $3 \times 3$ spin-one matrices as $\mathbf{S}=\left(S_{x}, S_{y}, S_{z}\right)$. The second term above describes hopping between second neighbors, and $\nu_{i j}=+1(-1)$ if the atom makes a left (right) turn to reach a second- 
neighbor site [14]. The spin components are decoupled in $\hat{\mathcal{H}}_{\mathrm{S} 1 \mathrm{KM}}$ : the spin-zero component is described by the nearest-neighbor graphene model with hopping $w$ while the spin- \pm 1 components are described by two Haldane models with opposite magnetic fields. We restrict our attention to $w>\sqrt{3}|\lambda|$ so that the single-particle state of lowest energy occurs at the centre of the Brillouin zone.

Next, we include on-site interactions that preserve spin rotation invariance [32-34]:

$$
\hat{\mathcal{H}}_{\mathrm{int}}=\sum_{i}\left[\frac{U}{2}\left(\hat{\boldsymbol{\Phi}}_{i}^{\dagger} \hat{\boldsymbol{\Phi}}_{i}\right)^{2}+\frac{U_{s}}{2}\left(\hat{\boldsymbol{\Phi}}_{i}^{\dagger} \mathbf{S} \hat{\boldsymbol{\Phi}}_{i}\right)^{2}\right]
$$

where $U$ and $U_{s}$ describe the magnitude of the density and spin interactions respectively. Finally, we introduce the standard quadratic Zeeman effect for spinor condensates [34]: $\hat{\mathcal{H}}_{\text {ext }}=q \sum_{i} \hat{\boldsymbol{\Phi}}_{i}^{\dagger}\left(S_{z}\right)^{2} \hat{\boldsymbol{\Phi}}_{i}$. While external magnetic fields will provide only positive values of $q$, microwave fields can be utilized to access both positive and negative quadratic Zeeman shifts [35]. Introducing a chemical potential $\mu$, the full Hamiltonian reads $\hat{\mathcal{H}}=\hat{\mathcal{H}}_{\mathrm{S} 1 \mathrm{KM}}+\hat{\mathcal{H}}_{\text {int }}+\hat{\mathcal{H}}_{\text {ext }}-\mu \sum_{i} \hat{\boldsymbol{\Phi}}_{i}^{\dagger} \hat{\boldsymbol{\Phi}}_{i}$. which is invariant under time reversal and global spin rotations about the $z$-axis, the importance of which will be addressed below.

In this work, we consider a quantum quench that abruptly changes the quadratic Zeeman energy $q$ from an initially large and positive value to a final value $q_{f}$. This form of quenching has been experimentally achieved in several experiments in the past decade (see [34] and references therein). The initial state is a coherent state with all bosons in a spatially uniform spin-zero state:

$$
\left|\Psi_{\text {in }}\right\rangle=e^{-\frac{1}{2} N_{p}} e^{\sqrt{\bar{n}} \sum_{i} \hat{\Phi}_{i, 0}^{\dagger}}|0\rangle
$$

where $N_{p}$ is the total atom number. There are no particles with $s_{z}= \pm 1$ in this state. This initial state is the mean-field superfluid ground state of the graphenelattice boson Hubbard model. A variational calculation shows that the average number of bosons per site, $\bar{n}$, is related to the chemical potential by $\mu=\bar{n} U-3 w$.

To investigate the ensuing dynamics after the quench, we consider small fluctuations of the Hamiltonian with $q=q_{f}$ around the initial state (3). Let $\hat{\boldsymbol{\phi}}_{i}=\hat{\boldsymbol{\Phi}}_{i}-$ $(0, \sqrt{\bar{n}}, 0)^{T}$ where $\hat{\phi}_{i}=\left(\hat{\phi}_{i, 1}, \hat{\phi}_{i, 0}, \hat{\phi}_{i,-1}\right)^{T}$. The Hamiltonian can be expanded to quadratic order in $\hat{\phi}_{i}$ as $\hat{\mathcal{H}}=\left\langle\Psi_{\text {in }}|\hat{\mathcal{H}}| \Psi_{\text {in }}\right\rangle+\hat{\mathcal{H}}_{B}$. One finds

$$
\begin{aligned}
\hat{\mathcal{H}}_{B} & =-w \sum_{\langle i j\rangle} \hat{\phi}_{i}^{\dagger} \hat{\phi}_{j}+i \lambda \sum_{\langle\langle i j\rangle\rangle} \nu_{i j} \hat{\phi}_{i}^{\dagger} S_{z} \hat{\phi}_{j}+\sum_{i} \hat{\phi}_{i}^{\dagger} M \hat{\phi}_{i} \\
& +\sum_{i}\left[\left(\frac{U \bar{n}}{2} \hat{\phi}_{i, 0} \hat{\phi}_{i, 0}+U_{s} \bar{n} \hat{\phi}_{i, 1} \hat{\phi}_{i,-1}\right)+\text { H.c. }\right]
\end{aligned}
$$

where $M=\operatorname{diag}\left(3 w+U_{s} \bar{n}+q_{f}, 3 w+U \bar{n}, 3 w+U_{s} \bar{n}+q_{f}\right)$ and $q_{f}$ is the quadratic Zeeman energy after the quench. The $S_{z}$-rotation symmetry of $\hat{\mathcal{H}}_{B}$ ensures that the spin\pm 1 components are decoupled from the spin- 0 components and hence the $(4)$ can be written as $\hat{\mathcal{H}}_{B}=\hat{\mathcal{H}}_{0}+$
$\hat{\mathcal{H}}_{ \pm 1}$. The Hamiltonian $\hat{\mathcal{H}}_{0}$ describes the dynamics of the spin-zero components and has no spin-orbit coupling. It is readily diagonalized by a Bogoliubov transformation. The resulting spectrum is stable and exhibits the usual linearly-dispersing phonon mode. From now on, we will focus on the spin- \pm 1 sector described by $\hat{\mathcal{H}}_{ \pm 1}$.

As we are primarily interested in the dynamics of the edge states of this model, we will focus on the strip geometry. Denoting the primitive lattice vectors of graphene as $\mathbf{a}_{1}$ and $\mathbf{a}_{2}$, we consider open (periodic) boundary conditions along the $\mathbf{a}_{1}\left(\mathbf{a}_{2}\right)$ direction. It is instructive to rewrite (4) in the eigenbasis of the non-interacting spinone Kane-Mele model (1) in this geometry. The spin- \pm 1 Hamiltonian becomes

$$
\begin{gathered}
\hat{\mathcal{H}}_{ \pm 1}=\sum_{k, \nu}\left[\left(\varepsilon_{k}^{(\nu)}-\Delta\right)\left(\hat{\alpha}_{k, \nu, 1}^{\dagger} \hat{\alpha}_{k, \nu, 1}+\hat{\alpha}_{-k, \nu,-1}^{\dagger} \hat{\alpha}_{-k, \nu,-1}\right)\right. \\
\left.+U_{s} \bar{n}\left(\hat{\alpha}_{k, \nu, 1} \hat{\alpha}_{-k, \nu,-1}+\text { H.c. }\right)\right] .
\end{gathered}
$$

Here, $\varepsilon_{k}^{(\nu)}$ are the single-particle energies of the Haldane model in the strip geometry, $k=\mathbf{k} \cdot \mathbf{a}_{2}$ is the momentum along the periodic direction, and $\hat{\alpha}_{k, \nu, m}$ annihilates a boson in the eigenbasis of (1). We have introduced $\Delta=-U_{s} \bar{n}-3 w-q_{f}$ which serves as the tuning parameter for our quench. Due to time-reversal symmetry and the spatial uniformity of the initial state, $\hat{\mathcal{H}}_{ \pm 1}$ can be separated into pairwise couplings between $(k, \nu, m)$ and $(-k, \nu,-m)$ modes which greatly simplifies the analysis.

Unlike $\hat{\mathcal{H}}_{0}, \hat{\mathcal{H}}_{ \pm 1}$ cannot in general be brought to diagonal form and may exhibit a dynamical instability. Therefore, we focus instead on the Heisenberg equations of motion: $i \partial_{t} \hat{\alpha}_{k, \nu, \pm 1}(t)=\left[\hat{\alpha}_{k, \nu, \pm 1}(t), \hat{\mathcal{H}}_{ \pm 1}\right]$ where $\hat{\alpha}_{k, \nu, \pm 1}(t)=e^{i \hat{\mathcal{H}}_{ \pm 1} t} \hat{\alpha}_{k, \nu, \pm 1} e^{-i \hat{\mathcal{H}}_{ \pm 1} t}$ and we have set $\hbar=1$. These can be solved to give

$$
\begin{aligned}
\hat{\alpha}_{k, \nu, 1}(t) & =A_{k, \nu}(t) \hat{\alpha}_{k, \nu, 1}+B_{k, \nu}(t) \hat{\alpha}_{-k, \nu,-1}^{\dagger}, \\
\hat{\alpha}_{-k, \nu,-1}(t) & =B_{k, \nu}(t) \hat{\alpha}_{k, \nu, 1}^{\dagger}+A_{k, \nu}(t) \hat{\alpha}_{-k, \nu,-1} .
\end{aligned}
$$

where $A_{k, \nu}(t)=\cos \left(E_{k}^{(\nu)} t\right)-i\left(\varepsilon_{k}^{(\nu)}-\Delta\right) \sin \left(E_{k}^{(\nu)} t\right) / E_{k}^{(\nu)}$ and $B_{k, \nu}(t)=-i U_{s} \bar{n} \sin \left(E_{k}^{(\nu)} t\right) / E_{k}^{(\nu)}$ with the Bogoliubov energies

$$
E_{k}^{(\nu)}=\sqrt{\left(\varepsilon_{k}^{(\nu)}-\Delta\right)^{2}-\left(U_{s} \bar{n}\right)^{2}} .
$$

For sufficiently low condensate depletion, the Bogoliubov Hamiltonian can be used to propagate the initial state (3) as $|\Psi(t)\rangle=e^{-i \hat{\mathcal{H}}_{B} t}\left|\Psi_{\text {in }}\right\rangle$. The solutions (6) can then be used to obtain the expectation value of bilinear operators:

$$
\begin{aligned}
& \left\langle\Psi(t)\left|\hat{\alpha}_{k, \nu, m}^{\dagger} \hat{\alpha}_{k^{\prime}, \nu^{\prime}, m^{\prime}}\right| \Psi(t)\right\rangle=\delta_{k, k^{\prime}} \delta_{\nu, \nu^{\prime}} \delta_{m, m^{\prime}}\left|B_{k, \nu}\right|^{2} \\
& \left\langle\Psi(t)\left|\hat{\alpha}_{k, \nu, m} \hat{\alpha}_{k^{\prime}, \nu^{\prime}, m^{\prime}}\right| \Psi(t)\right\rangle=\delta_{k,-k^{\prime}} \delta_{\nu, \nu^{\prime}} \delta_{m,-m^{\prime}} A_{k, \nu} B_{k, \nu}
\end{aligned}
$$

for spin $m= \pm 1$ components.

We now arrive at the central result of this work. If we tune the quench parameter $\Delta$ to satisfy

$$
\varepsilon_{k}^{(\nu)}-U_{s} \bar{n}<\Delta<\varepsilon_{k}^{(\nu)}+U_{s} \bar{n},
$$




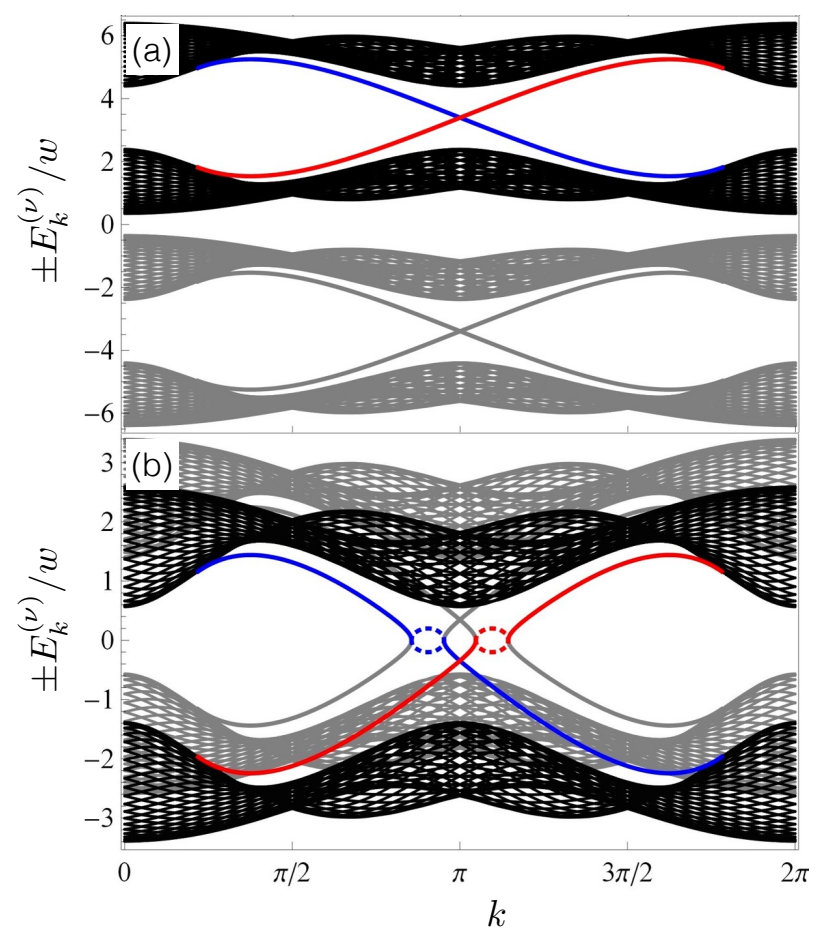

FIG. 1. (Color Online) The Bogoliubov energy spectrum $\pm E_{k}^{(\nu)}$ corresponding to (7) for the interacting spin-one KaneMele model in the strip geometry. Parameters in (a) are $U_{s} \bar{n}=0.2 w, \lambda=w / 2$ and $q_{f}=U_{s} \bar{n}$ which corresponds to a shallow quench with stable spectrum. Parameters in (b) are the same as (a) except $q_{f}=-3 w-3 U_{s} \bar{n}$ for which the bulk states are stable while the edge states experience an exponentially fast population growth. Gray curves indicate hole bands while black curves indicate bulk particle bands. Red and blue curves indicate edge states propagating in opposite directions. For clarity, edge states on only one side of the system are plotted (on the opposite side, the roles of the particle and hole edge bands are reversed). Imaginary parts of eigenvalues are given by dashed lines.

the Bogoliubov energies $E_{k}^{(\nu)}$ becomes imaginary signifying a dynamical instability for the $(k, \nu)$ mode. Physically, this provides exponentially fast population of the unstable modes. In other words, through (9), the quenching protocol gives a "window" (centered on $\Delta$ and of width $2 U_{s} \bar{n}$ ) of unstable modes in the spectrum $\varepsilon_{k}^{(\nu)}$.

To understand the instability criterion, we now discuss the single-particle eigenstates for the spin- \pm 1 components. As already mentioned, these are the eigenstates of the Haldane model with opposite fluxes for the two spin components. The bulk states form two bands of states separated by a band gap of $6 \sqrt{3} \lambda$ centered about zero [31]. In addition, the non-interacting spectrum exhibits topologically protected edge modes which exist within the bulk gap. In the Supplemental Material, we show that the dispersion of the edge states is

$$
\varepsilon_{k}^{\text {edge }}= \pm \frac{6 w \lambda \sin (k)}{\sqrt{w^{2}+16 \lambda^{2} \sin ^{2}\left(\frac{k}{2}\right)}}
$$

where spin- \pm 1 modes propagate in opposite directions. If we tune $\Delta$ to sit inside of the band gap, then, for sufficiently small $U_{s} \bar{n}$, we can achieve the intriguing situation where the bulk states are stable while the edge states experience exponentially fast population growth. A similar scheme for populating edge modes has been previously reported in [23]. Moreover, unlike [23], due to the tuneability of $\Delta$, the current scheme allows one to selectively populate states with particular momenta along the edge. From (7), we see that the most unstable modes occur at momenta for which $\varepsilon_{k}^{(\nu)}=\Delta$, so, for instance, when $\Delta=0$, edge modes with momenta $k=\pi$ will be populated most rapidly. Bulk and edge bands are shown in Fig. 1 for two particular quenches.

The symmetries (inversion, time reversal, and spin rotation) of the Bogoliubov Hamiltonian greatly simplify the above analysis. That is, given the single particle energies of the Haldane model, the above analysis essentially reduced to solving a two-mode problem. For more general couplings with less symmetry, it is easiest to proceed by solving the Bogoliubov-de Gennes (BdG) equations.

$$
\tau_{3} H_{k} v_{k, \nu}=E_{k}^{(\nu)} v_{k, \nu}
$$

where $H_{k}$ is the BdG Hamiltonian which, for our problem, can be directly determined from (4). For a system having length $N\left|\mathbf{a}_{1}\right|$ along the direction with open boundary conditions, $H_{k}$ is a $2 \mathcal{N} \times 2 \mathcal{N}$ dimensional matrix where $\mathcal{N}=6 N$ (the factor of 6 accounts for the spin and sublattice degrees of freedom) while $\tau_{3}=\sigma_{3} \otimes \mathbb{1}_{\mathcal{N} \times \mathcal{N}}$. The BdG Hamiltonian generically possesses a 'particlehole' symmetry which requires the eigenvalues to come in $\pm E_{k}^{(\nu)}$ pairs. For each pair of stable (real) eigenvalues, one member will have positive norm defined with the $\tau_{3}$ metric, $v_{k, \nu^{+}}^{\dagger} \tau_{3} v_{k, \nu^{+}}>0$, while the other will have negative norm $v_{k, \nu^{-}}^{\dagger} \tau_{3} v_{k, \nu^{-}}<0$. In analogy with BCS superconductors, we refer to bands composed of eigenstates with positive (negative) norms as 'particle' ('hole') bands, which are indicated in Fig. 1.

For stable systems, positive (negative) norm states correspond to positive (negative) eigenvalues $E_{k}^{(\nu)}$. This is not the case for unstable systems. In [36], the origin of a dynamical instability was traced to positive and negative norm states that become degenerate in the absence of pairing (non-particle number conserving) terms. Then, pairing terms generally lift such degeneracies and lead to complex Bogoliubov energies. This is precisely the mechanism leading to the unstable edge modes in our problem. On the other hand, as is evident from Fig. 1(b), one can have overlap between bulk particle and bulk hole bands which do not lead to dynamical instabilities. 
We interpret these bulk-band degeneracies as being protected by symmetries of the problem. Indeed, in the Supplemental Material we show that small contributions to the Hamiltonian that break time-reversal, inversion, or $S_{z}$ symmetry can hybridize the bulk particle and hole bands leading to bulk dynamical instabilities which would obscure the population growth of the edge modes. Such symmetries can be used to construct other bosonic models having unstable topological edge modes with stable bulk modes. However, for definiteness and due to experimental relevance [9], we focus on the interacting S1KM model in this work.

We now move on to discuss the physical consequences related to the quenching protocol. We first consider the number of particles excited into the spin- \pm 1 modes as a result of the quench. Using (8), we find

$$
\mathcal{N}_{ \pm 1}(t)=\sum_{i, m= \pm 1}\left\langle\Psi(t)\left|\hat{\phi}_{i, m}^{\dagger} \hat{\phi}_{i, m}\right| \Psi(t)\right\rangle=2 \sum_{k, \nu}\left|B_{k, \nu}\right|^{2} .
$$

For quenches satisfying (9) and chosen to select only edge modes for instability, the population growth in $\mathcal{N}_{ \pm 1}$ will be localized to the edges of the system. Keeping only unstable modes and linearizing the edge spectrum about $k=\pi$, for $\left|U_{s}\right| \bar{n} t \gg 1$ one finds

$$
\mathcal{N}_{ \pm 1}(t) \approx \sqrt{1+\frac{16 \lambda^{2}}{w^{2}}} \frac{N_{2}}{12 \lambda} \sqrt{\frac{\left|U_{s}\right| \bar{n}}{\pi t}} e^{2\left|U_{s}\right| \bar{n} t}
$$

where $N_{2}$ is the number of lattice sites along the $\mathbf{a}_{2}$ direction. Note that this expression is also valid for negative $U_{s}$, e.g. for ${ }^{87} \mathrm{Rb}$ atoms.

The quenching protocol is also expected to create a spin current along the edge. The continuity equation for the local spin moments $\hat{\boldsymbol{\Phi}}_{i}^{\dagger} S_{z} \hat{\boldsymbol{\Phi}}_{i}$ gives an expression for the spin current operator. At long wavelengths, the spin current operator along the edge is found to be [37]

$$
\hat{\mathcal{J}}_{k}^{\left(s_{z}\right)}=\frac{1}{N_{2}} \sum_{k^{\prime}, m= \pm 1} m \hat{\boldsymbol{\Phi}}_{k-\frac{k^{\prime}}{2}, m}^{\dagger} \partial_{k^{\prime}} H_{k^{\prime}}^{(m)} \hat{\boldsymbol{\Phi}}_{k+\frac{k^{\prime}}{2}, m}
$$

where $\hat{\mathbf{\Phi}}_{k, m}$ is a $2 N_{1}$-dimensional vector composed of annihilation operators for spin- $m$ bosons on sites in a unit cell of the strip geometry. $H_{k}^{(m)}$ is the noninteracting matrix Bloch Hamiltonian for spin component $m$ which can be directly determined from (1). We wish to evaluate the expectation value of this operator with the state $|\Psi(t)\rangle$. Writing $\hat{\mathcal{J}}_{k}^{\left(s_{z}\right)}$ in an eigenbasis of the non-interacting Hamiltonian, employing (8) and the Feynman-Hellman relation, we find the intuitive relation

$$
J^{\left(s_{z}\right)}(t) \equiv\left\langle\hat{\mathcal{J}}_{k=0}^{\left(s_{z}\right)}\right\rangle=\frac{2}{N_{2}} \sum_{k^{\prime}, \nu} \partial_{k^{\prime}} \varepsilon_{k^{\prime}}^{(\nu)}\left|B_{k^{\prime}, \nu}(t)\right|^{2}
$$

where the two spin components have contributed an equal amount. Additionally, the $k \neq 0$ components of
$\left\langle\hat{\mathcal{J}}_{k}^{\left(s_{z}\right)}\right\rangle$ vanish. Under the same conditions as were used for the evaluation of $\mathcal{N}_{ \pm 1}$, one finds

$$
J^{\left(s_{z}\right)}(t) \approx \frac{1}{4} \sqrt{\frac{\left|U_{s}\right| \bar{n}}{\pi t}} e^{2\left|U_{s}\right| \bar{n} t} .
$$

Before closing, we comment on the experimental feasibility of the above protocol. It is expected that quantum degeneracy in topological optical lattice systems will be reached in the near future with advances in experimental techniques. This will open new doors for exploring the non-equilibrium dynamics of topological band systems. In addition to the optical lattice, atoms are further confined in experiments by an overall (typically harmonic) trapping potential. Harmonic traps can obscure the edge states in the single-particle spectrum [38] of such systems. This problem can be surmounted by using box-shaped traps which are now available $[39,40]$. On the other hand, we expect many aspects of our results with open boundary conditions to be qualitatively correct for a harmonic trap provided the initial state is in the ThomasFermi regime. The reason is that the condensate will screen the trap potential, leading to an effective potential with sharp boundaries in the BdG equations for the edge excitations of interest. Indeed, the dynamics of a spinor condensate in a harmonic trap following a quench in the quadratic Zeeman field can be accurately modeled by an effective spherical-box potential [41]. Due to the sharp boundaries of the effective potential, we expect that well-defined edge states will continue to exist in this geometry. Finally, we note that our results will hold for either antiferromagnetic $\left(U_{s}>0\right)$ or ferromagnetic $\left(U_{s}<0\right)$ interactions and so are relevant for both ${ }^{87} \mathrm{Rb}$ and ${ }^{23} \mathrm{Na}$ condensates.

In summary, we have proposed a method whereby topological edge modes are populated exponentially fast through a quantum quench. Although edge states are typically unimportant for bosonic gases near equilibrium, we have shown that the non-equilibrium dynamics after a quench can be dominated by degrees of freedom localized on the boundary of the system. The growth of the edge modes will be limited at longer times by interactions not captured in the Bogoliubov theory. The long-time decay mechanism of the dynamically populated edge modes will also be due to these interaction terms.

This quenching protocol provides a means of collecting cold atoms coherently in a quasi-one-dimensional structure without the need for extra trapping lasers. Though this work focused on ultracold atoms, it will also be worthwhile to consider the parallels with photonic lattices where pairing terms can be generated by nonlinear optical methods.

We are grateful for support from the Schrodinger Scholarship Scheme at Imperial College London (B. G.); the European Union's Seventh Framework Programme for research, technological development, and demonstration under Grant No. PCIG-GA-2013-631002 and the As- 
pen Center for Physics under Grant No. PHYS-1066293 (R. B.).

\section{SUPPLEMENTAL MATERIAL}

\section{Derivation of the edge-state dispersion}

Our aim is to analyze the single-particle edge states of $\hat{\mathcal{H}}_{\mathrm{S} 1 \mathrm{KM}}$ from the main text. Note that since this Hamiltonian is invariant under spin rotations about the $z$ axis, we can consider each spin component separately. We first consider the Hamiltonian describing $m=1$ spin component, which is the Haldane model. The primitive lattice vectors, as shown in Fig. 2 are $\mathbf{a}_{1}=\left(\frac{\sqrt{3}}{2}, \frac{1}{2}\right)$ and $\mathbf{a}_{2}=\left(\frac{\sqrt{3}}{2},-\frac{1}{2}\right)$ where we have set the lattice constant to unity. We impose periodic boundary conditions along the $\mathbf{a}_{2}$-direction and take the system to be semi-infinite along the $\mathbf{a}_{1}$-direction. The single particle energies $\varepsilon_{k}$ are found by solving the following Schrodinger equation

$$
R_{k} \boldsymbol{\psi}_{n, k}+V_{k} \boldsymbol{\psi}_{n+1, k}+V_{k}^{\dagger} \boldsymbol{\psi}_{n-1, k}=\varepsilon_{k} \boldsymbol{\psi}_{n, k}
$$

for $n>1$ and

$$
R_{k} \boldsymbol{\psi}_{n, k}+V_{k} \boldsymbol{\psi}_{n+1, k}=\varepsilon_{k} \boldsymbol{\psi}_{n, k}
$$

for $n=1$. Here $\boldsymbol{\psi}_{n, k}=\left(\psi_{n, k}^{(A)}, \psi_{n, k}^{(B)}\right)^{T}$ is a two-component vector where $n$ labels unit cell and $A, B$ label sublattice (see Fig. 2). $R_{k}$ and $V_{k}$ are $2 \times 2$ matrices given by

$$
R_{k}=\left(\begin{array}{cc}
-2 \lambda \sin (k) & -w\left(1+e^{-i k}\right) \\
-w\left(1+e^{i k}\right) & 2 \lambda \sin (k)
\end{array}\right)
$$

and

$$
V_{k}=\left(\begin{array}{cc}
-i \lambda\left(1-e^{-i k}\right) & 0 \\
-w & i \lambda\left(1-e^{-i k}\right)
\end{array}\right) .
$$

The Schrodinger equation written above and its constituent matrices directly follow from the non-interacting Hamiltonian $\hat{\mathcal{H}}_{\mathrm{S} 1 \mathrm{KM}}$.

We search for a solution of the form

$$
\left.\boldsymbol{\psi}_{n, k}=\frac{1}{\kappa_{1, k}-\kappa_{2, k}}\left[\left(\kappa_{1, k}\right)^{n}-\left(\kappa_{2, k}\right)^{n}\right)\right] \boldsymbol{\psi}_{1, k}
$$

where we require that $\left|\kappa_{1, k}\right|,\left|\kappa_{2, k}\right|<1$ so that the solution decays into the bulk. Inserting this into the Schrodinger equation, we find $\left(R_{k}+V_{k} \kappa_{1, k}+\right.$ $\left.\frac{1}{\kappa_{1, k}} V_{k}^{\dagger}\right) \boldsymbol{\psi}_{1, k}=\varepsilon_{k} \boldsymbol{\psi}_{1, k}$ and $\left(R_{k}+V_{k} \kappa_{2, k}+\frac{1}{\kappa_{2, k}} V_{k}^{\dagger}\right) \boldsymbol{\psi}_{1, k}=$ $\varepsilon_{k} \boldsymbol{\psi}_{1, k}$ which can be rearranged to give the following two equations:

$$
\begin{aligned}
V_{k}^{-1} V_{k}^{\dagger} \boldsymbol{\psi}_{1, k} & =\kappa_{1, k} \kappa_{2, k} \boldsymbol{\psi}_{1}, \\
\left(R_{k}+\left(\kappa_{1, k}+\kappa_{2, k}\right) V_{k}\right) \boldsymbol{\psi}_{1, k} & =\varepsilon_{k} \boldsymbol{\psi}_{1, k} .
\end{aligned}
$$

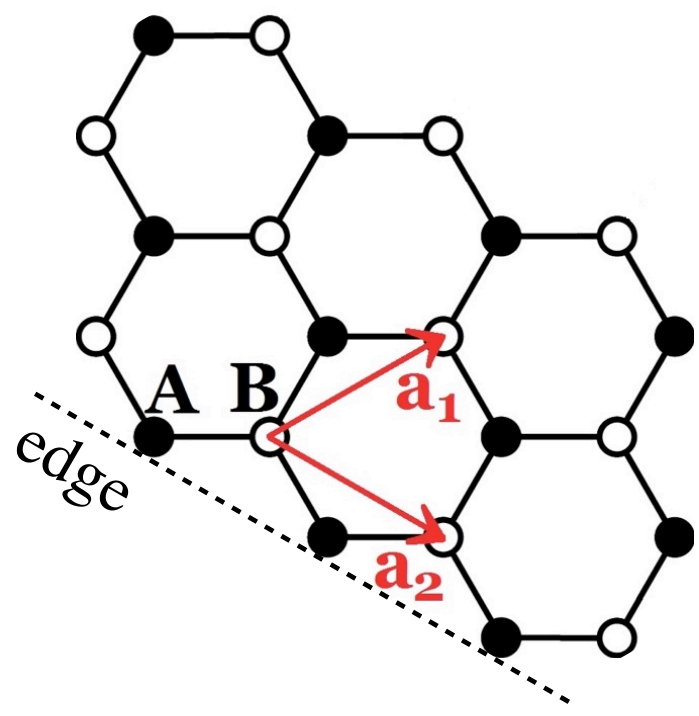

FIG. 2. A portion of the hexagonal lattice used for the spinone Kane-Mele Hamiltonian. Triangular sublattice sites $A$ and $B$ are labelled by closed and open circles respectively. The lattice basis vectors are given by $\mathbf{a}_{1}$ and $\mathbf{a}_{2}$.

These equations can be directly solved to determine $\boldsymbol{\psi}_{1}$ (up to a prefactor), $\kappa_{1}, \kappa_{2}$, and $\varepsilon_{k}$. In particular, for the edge dispersion we find

$$
\varepsilon_{k}=-\frac{6 w \lambda \sin (k)}{\sqrt{w^{2}+16 \lambda^{2} \sin ^{2}\left(\frac{k}{2}\right)}} .
$$

The edge mode energies for the $m=0$ and $m=-1$ spin components can be found with the replacements $\lambda \rightarrow 0$ and $\lambda \rightarrow-\lambda$ respectively.

\section{Symmetry protection of bulk instabilities}

We now show that small perturbations to the Hamiltonian $\hat{\mathcal{H}}_{B}$ (Eq. 4 from the main text) that break time reversal symmetry (TRS), inversion symmetry (IS), or $S_{z}$ symmetry can lead to the emergence of dynamical instabilities coming from the hybridization of bulk modes in the particle-hole picture. Below we give three terms that we add to the Hamiltonian $\hat{\mathcal{H}}_{B}$ which break either TRS, IS, or $S_{z}$ symmetry while preserving the other two. To break $S_{z}$ symmetry we consider the contribution of same spin-component pairing terms

$$
\hat{V}_{S_{z}}=\delta \sum_{i}\left(\hat{\phi}_{i, 1} \hat{\phi}_{i, 1}+\hat{\phi}_{i,-1} \hat{\phi}_{i,-1}+\text { H.c. }\right)
$$

where $\delta$ is taken to be a real and small in comparison to other energy scales of the problem. This term preserves TRS and IS. Inversion symmetry can be broken, while keeping the TRS and $S_{z}$ symmetry intact, by adding a 


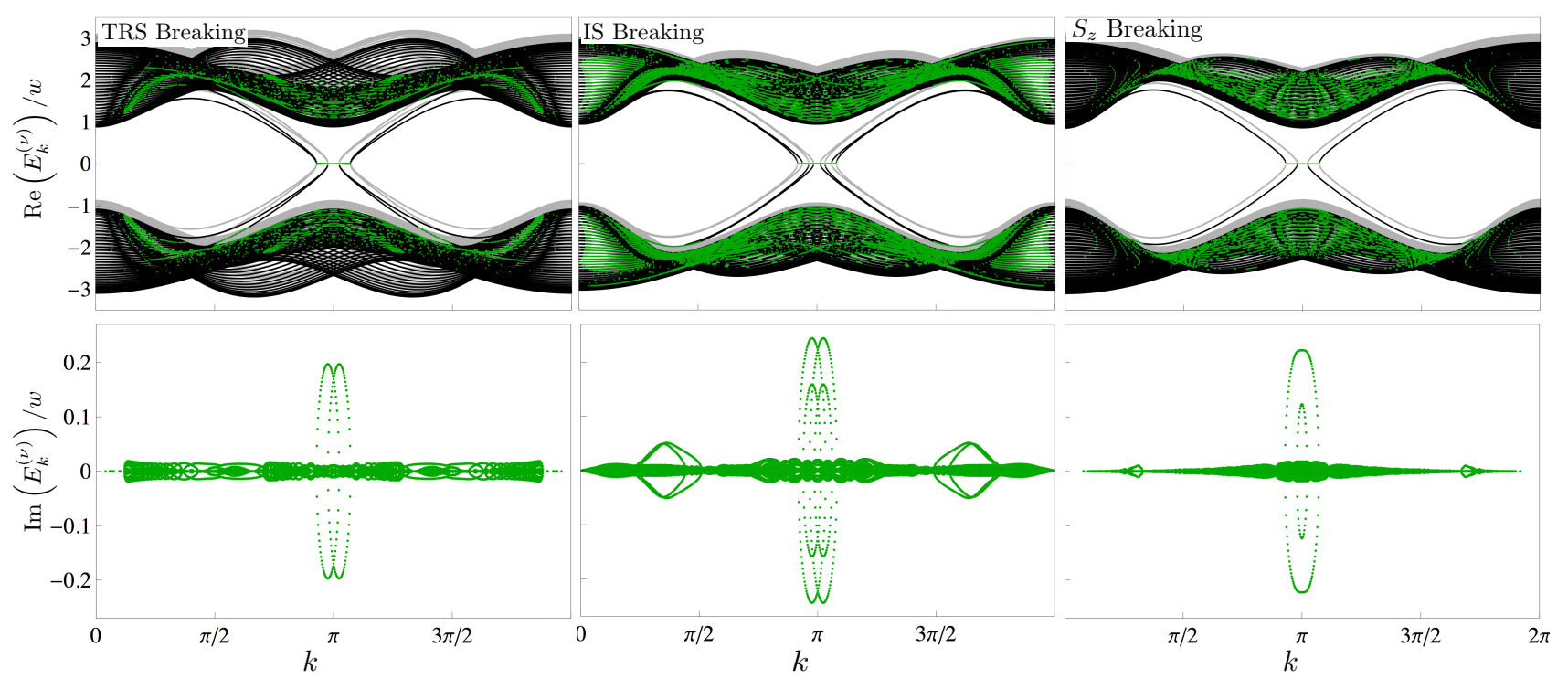

FIG. 3. Bogoliubov energy spectrum $E_{k}^{(\nu)}$ for the interacting spin-one Kane-Mele model with broken time-reversal, inversion, and $S_{z}$ symmetries. Parameters are chosen to be $U_{s} \bar{n}=0.2 w, \lambda=w / 2, q_{f}=-3 w-3 U_{s} \bar{n} / 2$, and $\delta=0.095 w$. Gray curves indicate hole bands while black curves indicate bulk bands. The energy values corresponding to dynamically unstable modes (with finite imaginary component) are colored in green. For clarity, modes from the zero spin component (which is always stable) are excluded from these plots.

staggered pairing between the two triangular sublattices $A$ and $B$ (Fig. 2):

$$
\hat{V}_{I}=\delta \sum_{n}\left(\hat{\phi}_{n, 1}^{(A)} \hat{\phi}_{n,-1}^{(A)}-\hat{\phi}_{n, 1}^{(B)} \hat{\phi}_{n,-1}^{(B)}+\text { H.c. }\right)
$$

where $n$ labels unit cells. Finally, we consider a term that changes sign under time reversal, but preserves IS and $S_{z}$ symmetry:

$$
\hat{V}_{T}=i \delta \sum_{\langle\langle i j\rangle\rangle} \nu_{i j} \hat{\boldsymbol{\phi}}_{i}^{\dagger} S_{z}^{2} \hat{\boldsymbol{\phi}}_{j}
$$

In Fig. 3 we show the real and imaginary parts of Bogoliubov energy spectrum computed numerically after adding each of these terms to the Hamiltonian $\hat{\mathcal{H}}_{B}$. In addition to edge dynamical instabilities discussed in the main text, some bulk bands acquire a small imaginary part in the energy spectrum. This effect is amplified with the increase of $\delta$ and disappears in the absence of the symmetry breaking terms.

We now move on to discuss a slightly more stringent sufficient condition for the absence of bulk instabilities. We impose the condition of TRS and $S_{z}$ symmetry, and further require that the mean-field density (used to derive the Bogoliubov Hamiltonian) is spatially uniform. With these requirements, it can be shown that $\left(\tau_{3} H_{k}\right)^{2}$ is a Hermitian matrix where $H_{k}$ is the BdG Hamiltonian introduced in Eq. (11) from the main text. This implies that the eigenvalues of $\left(\tau_{3} H_{k}\right)^{2}$, namely $\left(E_{k}^{(\nu)}\right)^{2}$, will be real numbers and so $E_{k}^{(\nu)}$ will either be pure real or pure imaginary. This means that an imaginary part in the energy spectrum will arise only in the region of overlap of particle and hole bands with vanishing real part of the energy. When the zero value of the real energy spectrum is situated in the gapped region, no dynamical instability is induced except in the crossing edge states. In this sense, these symmetries protect the bulk bands from becoming dynamically unstable. While the model we consider in the main text possesses these symmetries, it is possible that less stringent conditions exist.

[1] D. J. Thouless, M. Kohmoto, M. P. Nightingale, and M. den Nijs, Phys. Rev. Lett. 49, 405 (1982).

[2] M. Z. Hasan and C. L. Kane, Rev. Mod. Phys. 82, 3045 (2010).

[3] X.-L. Qi and S.-C. Zhang, Rev. Mod. Phys. 83, 1057 (2011).

[4] N. Goldman, G. Juzeliūnas, P. Ohberg, and I. B. Spielman, Reports on progress in physics. Physical Society (Great Britain) 77, 126401 (2014).

[5] M. Atala, M. Aidelsburger, J. T. Barreiro, D. Abanin, T. Kitagawa, E. Demler, and I. Bloch, Nature Physics 9, 795 (2013).

[6] R. A. Williams, S. Al-Assam, and C. J. Foot, Phys. Rev. Lett. 104, 050404 (2010).

[7] M. Aidelsburger, M. Atala, M. Lohse, J. T. Barreiro, B. Paredes, and I. Bloch, Phys. Rev. Lett. 111, 185301 
(2013).

[8] H. Miyake, G. A. Siviloglou, C. J. Kennedy, W. C. Burton, and W. Ketterle, Phys. Rev. Lett. 111, 185302 (2013).

[9] G. Jotzu, M. Messer, R. Desbuquois, M. Lebrat, T. Uehlinger, D. Greif, and T. Esslinger, Nature 515, 237 (2014).

[10] F. D. M. Haldane and S. Raghu, Phys. Rev. Lett. 100, 013904 (2008).

[11] Z. Wang, Y. Chong, J. Joannopoulos, and M. Soljačić, Nature 461, 772 (2009).

[12] M. Hafezi, E. A. Demler, M. D. Lukin, and J. M. Taylor, Nature Physics 7, 907 (2011).

[13] M. C. Rechtsman, J. M. Zeuner, Y. Plotnik, Y. Lumer, D. Podolsky, F. Dreisow, S. Nolte, M. Segev, and A. Szameit, Nature 496, 196 (2013).

[14] C. L. Kane and E. J. Mele, Phys. Rev. Lett. 95, 146802 (2005).

[15] M. Killi, S. Trotzky, and A. Paramekanti, Phys. Rev. A 86, 063632 (2012).

[16] M. S. Foster, M. Dzero, V. Gurarie, and E. A. Yuzbashyan, Phys. Rev. B 88, 104511 (2013).

[17] G. Kells, D. Sen, J. K. Slingerland, and S. Vishveshwara, Phys. Rev. B 89, 235130 (2014).

[18] P. Hauke, M. Lewenstein, and A. Eckardt, Phys. Rev. Lett. 113, 045303 (2014).

[19] R. Vasseur, J. P. Dahlhaus, and J. E. Moore, Phys. Rev. X 4, 041007 (2014).

[20] P. D. Sacramento, Phys. Rev. E 90, 032138 (2014).

[21] E. Eriksson, A. Zazunov, P. Sodano, and R. Egger, Phys. Rev. B 91, 064501 (2015).

[22] M. Caio, N. Cooper, and M. Bhaseen, arXiv preprint arXiv:1504.01910 (2015).

[23] R. Barnett, Phys. Rev. A 88, 063631 (2013).

[24] A. J. Heeger, S. Kivelson, J. R. Schrieffer, and W. P. Su,
Rev. Mod. Phys. 60, 781 (1988).

[25] C. Poli, M. Bellec, U. Kuhl, F. Mortessagne, and H. Schomerus, Nature Communications 6, 6710 (2015).

[26] H. Schomerus, Opt. Lett. 38, 1912 (2013).

[27] A. P. Schnyder, S. Ryu, A. Furusaki, and A. W. W. Ludwig, Phys. Rev. B 78, 195125 (2008).

[28] A. Kitaev, AIP Conf. Proc. 1134 (2009).

[29] R. Shindou, R. Matsumoto, S. Murakami, and J.-i. Ohe, Phys. Rev. B 87, 174427 (2013).

[30] G. Engelhardt and T. Brandes, Phys. Rev. A 91, 053621 (2015).

[31] F. D. M. Haldane, Phys. Rev. Lett. 61, 2015 (1988).

[32] T.-L. Ho, Phys. Rev. Lett. 81, 742 (1998).

[33] T. Ohmi and K. Machida, Journal of the Physical Society of Japan 67, 1822 (1998).

[34] D. M. Stamper-Kurn and M. Ueda, Rev. Mod. Phys. 85, 1191 (2013).

[35] F. Gerbier, A. Widera, S. Fölling, O. Mandel, and I. Bloch, Phys. Rev. A 73, 041602 (2006).

[36] Y. Nakamura, M. Mine, M. Okumura, and Y. Yamanaka, Phys. Rev. A 77, 043601 (2008).

[37] B. A. Bernevig, Topological insulators and topological superconductors (Princeton University Press, 2013).

[38] M. Buchhold, D. Cocks, and W. Hofstetter, Phys. Rev. A 85, 063614 (2012).

[39] A. L. Gaunt, T. F. Schmidutz, I. Gotlibovych, R. P. Smith, and Z. Hadzibabic, Phys. Rev. Lett. 110, 200406 (2013).

[40] L. Chomaz, L. Corman, T. Bienaimé, R. Desbuquois, C. Weitenberg, S. Nascimbène, J. Beugnon, and J. Dalibard, Nat. Commun. 6, 6162 (2015).

[41] M. Scherer, B. Lücke, G. Gebreyesus, O. Topic, F. Deuretzbacher, W. Ertmer, L. Santos, J. J. Arlt, and C. Klempt, Phys. Rev. Lett. 105, 135302 (2010). 